\title{
Erratum: Semantic Incompleteness of Quantum Physics ${ }^{1}$
}

\section{Claudio Garola ${ }^{2}$}

The following changes should be made in this paper:

Page 811, line 29. Insert

(vi) Auxiliary signs (.), $/$.

Page 817, line 2. Delete

(Garola, 1991b).

Page 819, line 23. The equation

$$
A_{r}=\left(\pi_{r} x\right)(S(x) \rightarrow E(x))
$$

should be replaced by the equation

$$
A_{r}=\left(\pi_{r} x\right)(E(x) / S(x))
$$

Page 820 , line 18 . The formula

$$
\left(\pi_{\lambda_{k}} x\right)\left(S(x) \rightarrow E_{s_{k}}(x)\right)
$$

should be replaced by the formula

$$
\left(\pi_{\lambda_{k}} x\right)\left(E_{s_{k}}(x) / S(x)\right)
$$

Page 820, line 39. The equation

$$
A_{r}=\left(\pi_{r} x\right)(S(x) \rightarrow E(x))
$$

should be replaced by the equation

$$
A_{r}=\left(\pi_{r} x\right)(E(x) / S(x))
$$

Page 821 , line 2 in footnote 6 . The equation

$$
A_{r}=\left(\pi_{r} x\right)(S(x) \rightarrow E(x))
$$

should be replaced by the equation

$$
A_{r}=\left(\pi_{r} x\right)(E(x) / S(x))
$$

'This paper appeared in International Journal of Theoretical Physics, 31(5), 809-824.

${ }^{2}$ Dipartimento di Fisica, Università degli Studi di Lecce, 73100 Lecce, Italy. 